\title{
Management of Cervical Kyphosis in Larsen Syndrome: A Case Report
}

\author{
Ebrahim Ameri, ${ }^{1}$ Farshad Nekoui, ${ }^{1,}$ Abouzar Azizi, ${ }^{1}$ and Saeid Sabbaghan ${ }^{1}$ \\ ${ }^{1}$ Bone and Joint Reconstruction Research Center, Shafa Orthopedic Hospital, Iran University of Medical Sciences, Tehran, IR Iran \\ "Corresponding author: Farshad Nekoui, Bone and Joint Reconstruction Research Center, Shafa Orthopedic Hospital, Tehran, IR Iran. Tel: +98-2133542000-8, Fax: \\ +98-2133542020, E-mail: nekoui.far@gmail.com
}

Received 2016 April 15; Revised 2016 June 21; Accepted 2016 September 13.

\begin{abstract}
Introduction: Larsen syndrome is a congenital skeletal disorder manifested by several facial, ligamentous and spinal complications. Cervical kyphosis is one of the serious manifestations of the Larsen syndrome. However, there is no consensus regarding the best procedure of cervical kyphosis management in these patients.

Case Presentation: A1-year-old boy with the diagnosis of the Larsen syndrome was admitted to our hospital and undergone several corrective surgeries for knee, hip and foot deformities. At the age of 2 years, scoliosis was diagnosed and surgically managed. At the same time, cervical kyphosis was observed and monitored until the symptoms of neurological deficit due to cord compression led to the correction of cervical Kyphosis at the age of 4.5 years. Accordingly, an anterior/posterior (360 degree) cervical spinal fusion surgery was performed. Subsequently, cervicothoracic fusion was performed to correct cervicothoracic instability. No neurological complications were reported afterward.

Conclusions: In spite of existing controversy around the best method of cervical kyphosis management in Larsen syndrome's patients older than 2-year old, anterior release and posterior fixation followed by anterior spinal fusion and strut grafting led to the satisfactory result in our case.
\end{abstract}

Keywords: Larsen Syndrome, Cervical Kyphosis, Anterior/Posterior Spinal Fusion

\section{Introduction}

Larsen syndrome is a congenital skeletal dysplasia manifested mainly by anomalous facial features, ligamentous laxity, multiple joint dislocations plus foot, hand and spinal deformity (1). Among various spinal deformities of the Larsen syndrome including spina bifda, hypoplasia of vertebral bodies, thoracolumbar scoliosis and etc, cervical kyphosis is of particular importance due to the possibility of fatal impingement of the apex of the deformity on the spinal cord (1-3). Cervical kyphosis is a common manifestation of the Larsen syndrome. Therefore, the correction of cervical kyphosis is a crucial part of the Larsen syndrome's management, which demands close monitoring of the patients in order to detect the evidences of neurological symptoms. Cervical kyphosis can be easily detected through the lateral view of the cervical radiographs.

Different centers apply different approaches to manage the cervical kyphosis of the Larsen syndrome (4). However, most of these approaches are not adequate and the literature review indicates that about half of the reported cases have failed to improve and require additional operations (4). Reporting new cases and their management approach could finally result in an improved management of this complication, which elevates the quality of life of the suffering patients (4). In this regard, we here report the management approach of a cervical kyphosis in a patient with the Larsen syndrome who has been referred to our center and compare its result with the result of the different approaches implemented in the other centers.

\section{Case Presentation}

A boy, with several congenital deformities including bilateral hip, knee and ankle deformity, diagnosed with the Larsen disease referred to our hospital to get the required treatment. The patients had limited knee and hip joint movement in addition to the hearing loss, which has already been corrected using hearing aids (Figure 1).

When the patient was 1-year old, we managed the complications through several deformity corrective operations including knee, hip and foot corrections. At the age of 2 years, the patient was operated again in order to correct the observed thoracic scoliosis with the implementation of dual growing rods (Figure 2). Subsequently, the rods were lengthened every 6 months, totally 4 times.

At the same time, cervical kyphosis was observed and monitored until the symptoms of the upper nervous system involvement including frequent fallings, paresis and 

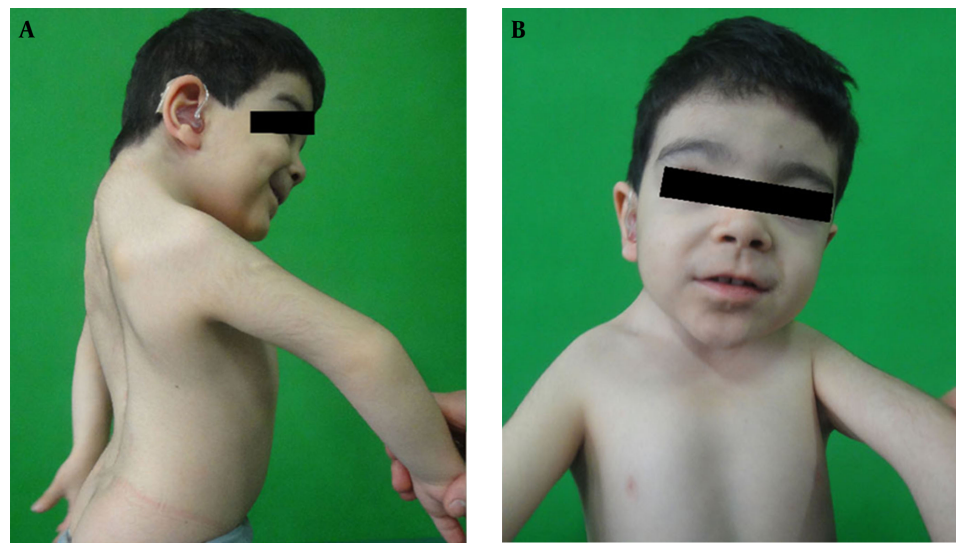

Figure 1. A, Skeletal Deformity and B, Facial Dysmorphy of the Larsen Syndrome Case
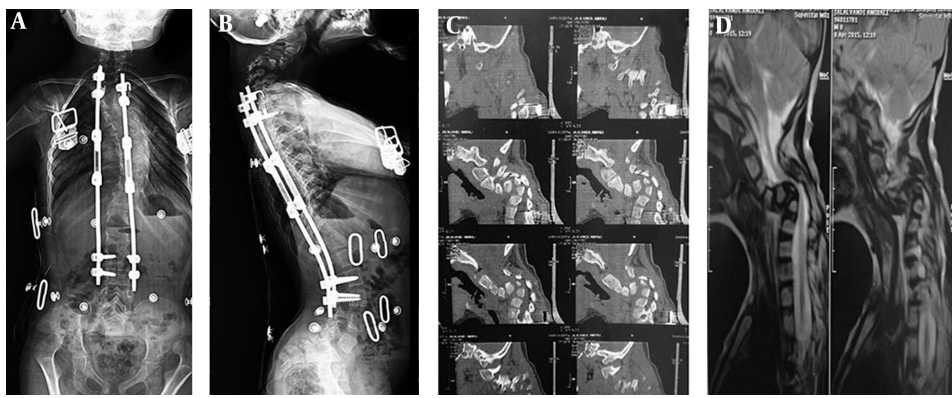

Figure 2. A, AP (Anterior/Posterior) and B, Lateral View of Thoracic Dual Growing Rod Implementation; Cervical Spine CT Scan Showing Severe Impingement on the C, Spinal Cord Cervical Spine T2-Weighted MRI Confirming the Result of D, the CT Scan

spasticity followed by confirmatory CT scan and MRI of cervical spine proved the impinging of the spinal cord by cervical kyphosis (Figure 2). As a result, anterior/posterior (360 degree) cervical spinal fusion plus halo vest implementation was performed when the patient was 4.5 years old, which resulted in the elimination of neurological symptoms (Figure 3). In this regard, through a vertical standard incision in the anterior neck we first performed the cervical corpectomy surgery (at C4 and C5), followed by fibular strut allograft application. Subsequently, the C3C6 posterior rod-hook fixation was implemented. However, after the posterior approach the anterior allograft was failed. In consequence, a fibular strut graft was adjusted again for anterior cervical fusion. The halo vest was removed 4 months later.

During the follow-up sessions, cervico-thoracic instability and gradual kyphosis was detected clinically and radiologically. Initiation of neurological symptoms led to the subsequent C6-T2 cervico-thoracic fusion surgery (Figure 4). No other complaints have been reported after the last surgery.
The patient's parent was informed that the data of the case would be submitted for publication, and provided written consent. In addition, Iran University of Medical Sciences' IRB has reviewed and approved the Waiver of authorization for the use of protected health information (PHI) for research purposes for this report.

\section{Discussion}

Cervical kyphosis is a life-threatening manifestation of the Larsen syndrome due to the high risk of progression of the kyphosis, which could lead to the paralysis or death. Johnson et al. reported that from 9 patients with the Larsen syndrome referred to their center, 5 were diagnosed with cervical kyphosis, which was the result of marked hypoplasia of 1 or 2 vertebral bodies at the apex of kyphosis (2). It is recommended to closely monitor cervical kyphosis in patients with the Larsen syndrome in order to prevent its serious injuries (1). Various therapeutic options including conservative observation and surgical correction have been 

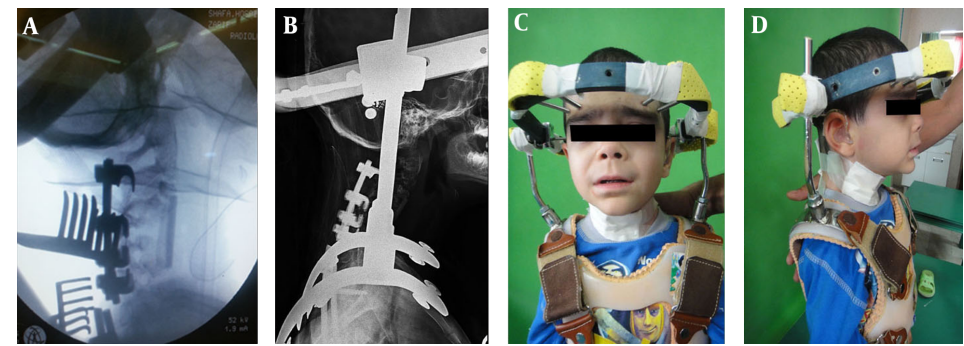

Figure 3. A, AP and B, and Lateral Radiograph of Anterior/Posterior Spinal Fusion Plus Halo Vest Implementation; the Patient's C, Anterior and D, Lateral View Following Anterior/Posterior Spinal Fusion and Halo Vest Implementation
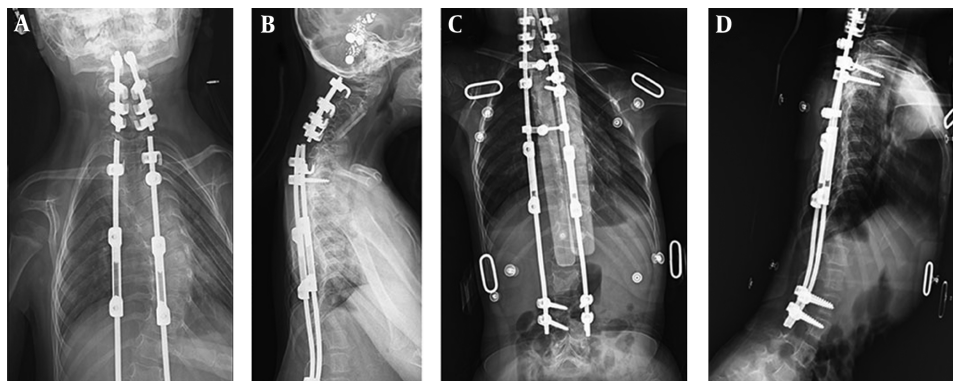

Figure 4. AP and Lateral Radiographs A, B, Before; C, D, After (the Cervicothoracic Fusion Procedure

recommended for patients with the presentation of cervical spine pathophysiology. However, there is no consensus regarding the best surgical approach, timing of the correction and pre- or post-operative bracing of cervical kyphosis in the Larsen syndrome (5). In addition, some authors have recommended that the orthopedic spine surgeons must be aware of the potential risk factors of disease before making the surgery decision. The rare complications of the Larsen syndrome including hearing loss and laryngomalacia are reported as the risk factors of the surgery in these patients, which should be cautiously evaluated in order to avoid critical situations during the surgery (6). Since hearing loss, as a potential risk factor of the surgery, was observed in our case, we carefully evaluated patient's condition, preoperatively, to minimize the potential risks of surgery. This included the ENT (Ear, Nose, Throat), anesthesia and pediatric consultations.

According to previous reports, in 8 out of 15 patients with the Larsen syndrome the cervical kyphosis was diagnosed at the age of less than 2 years (4). In our case, the development of patient's cervical kyphosis was regularly monitored and was diagnosed at the age of 4 . Posterior spinal fusion is the recommended approach in young patients. According to a former literature review, among 9 patients indicating neurological deficits of the Larsen syndrome, posterior spinal fusion was the first surgical ap- proach in 8 patients (4). It has been indicated that posterior spinal fusion provides spinal stability through permitting the growth of anterior spinal elements to spontaneously correct the kyphosis after surgery (4). However, the success rate of posterior spinal fusion is $66 \%$ in patients younger than 2 years old and 50\% in those older $(7,8)$. As a result, recent studies recommend the posterior spinal fusion surgery along with anterior correction in patient older than 2-year- old $(4,9)$. As our patient was four-yearold at the time of the diagnosis of cervical kyphosis, we also performed both anterior correction and posterior spinal fusion surgery. As aforementioned, a considerable number of patients treated with posterior spinal fusion needed additional surgeries. However, in a nearly 1 year follow-up period, no complication of cervical kyphosis has been observed in our patient and anterio-posterior fusion was confirmed in the radiographs of follow-up sessions. In conclusion, we believe that the simultaneous antero-posterior spinal fusion surgery could be an appropriate approach in the management of cervical kyphosis in the Larsen syndrome's patients older than 2-year-old. To this aim, we suggest anterior release and posterior fixation followed by anterior spinal fusion and strut grafting could lead to the satisfactory outcome in similar cases. 


\section{Footnote}

Authors' Contribution: Ebrahim Ameri and Farshad Nekoui: diagnosis and treatment; Ebrahim Ameri, Farshad Nekoui, Abouzar Azizi, and Saeid Sabbaghan: manuscript preparation and revision.

\section{References}

1. Latta RJ, Graham CB, Aase J, Scham SM, Smith DW. Larsen's syndrome: a skeletal dysplasia with multiple joint dislocations and unusual facies. J Pediatr. 1971;78(2):291-8. [PubMed: 5539773].

2. Johnston CE, Birch G, Daniels JL. Cervical kyphosis in patients who have Larsen syndrome. J Bone Joint Surg Am. 1996;78(4):538-45.

3. Larsen LJ, Schottstaedt ER, Bost FC. Multiple congenital dislocations associated with characteristic facial abnormality. $J$ Pediatr. 1950;37(4):574-81. [PubMed: 14779259].
4. Angsanuntsukh C, Tomlinson LA, Dormans JP. Review of cervical kyphosis in Larsen syndrome. Spine Deform. 2012;1.

5. Bowen JR, Ortega K, Ray S, Macewen GD. Spinal deformities in Larsen's syndrome. Clini Orthopaed Relat Res. 1985;197:159-63.

6. Karakas K, Percin EF, Percin S. Surgical risk factors in Larsen's syndrome. Acta Orthop Belg. 2000;66(5):495-8. [PubMed: 11196375].

7. Sakaura H, Matsuoka T, Iwasaki M, Yonenobu K, Yoshikawa H. Surgical treatment of cervical kyphosis in Larsen syndrome: report of 3 cases and review of the literature. Spine (Phila Pa 1976). 2007;32(1):E39-44. doi: 10.1097/01.brs.0000250103.88392.8e. [PubMed: 17202879].

8. Luk KD, Yip DK. Congenital anteroposterior spinal dissociation in Larsen's Syndrome: report on two operated cases with long-term follow-up. Spine (Phila Pa 1976). 2002;27(12):E296-300. [PubMed: 12065992]

9. Madera M, Crawford A, Mangano FT. Management of severe cervical kyphosis in a patient with Larsen syndrome. J Neur. 2008;1(4):320-4. 